Bull. Mater. Sci., Vol. 20, No. 6, September 1997, pp. 885-900. (C Printed in India.

\title{
Analytical and numerical studies of deformation behaviour at microscopic scale
}

\author{
N RAMAKRISHNAN \\ Computer Simulation Centre, Defence Metallurgical Research Laboratory, Kanchanbagh, \\ Hyderabad 500058 , India
}

\begin{abstract}
The paper presents an overview of the analytical methods as well as finite element method employed by the author in a few earlier investigations pertaining to modelling and simulation of deformation at microscopic scale. The following case-studies are considered for illustrations: deformation of a set of powder particles during hot isostatic pressing; effective properties of a typical particulate metal matrix composite and porous material; constitutive behaviour of a material exhibiting transformation-induced plasticity; shear band formation in polycrystalline material.

The paper describes certain generalized techniques for constructing the microstructural geometries, assigning material properties and imposing boundary conditions. The concept of generating two-phase geometries using a master mesh and the generalized plane strain approach to handle two-dimensional approximations used in the above studies are also reviewed. In contrast to the commonly employed unit cell models based on certain regular geometries, the present method uses the actually observed microstructural geometries. This method accommodates more realistic and complex conditions compared to those supported by the well-explored analytical methods. Although, only homogeneous and isotropic systems have been discussed in this paper, this method can be easily extended to inhomogeneous and anisotropic cases as well. In general, the technique is emerging as a suitable numerical tool for designing materials for specific applications.
\end{abstract}

Keywords. FEM; deformation; microstructure.

\section{Introduction}

There are a number of numerical as well as analytical methods available today to study the micro-mechanics of materials. The analytical methods are attractive since they offer better physical insight, but are generally restricted to simple geometries and any attempt to extend them to complex ones invariably leads to mathematical intractability. A majority of the analytical models available today are essentially the variants of composite sphere model (Hashin 1962; Hashin and Shtrikman 1963), self consistent model (Budiansky 1965; Hill 1965), differential model (McLaughlin 1977; Norris 1985; Zimmerman 1991) or Mori-Tanaka model (Mori and Tanaka 1973; Benvensite 1987). A comprehensive overview of the analytical methods is presented by Christensen (1990). Many of the numerical and analytical models (Mckenzie and Shuttleworth 1948; Kovalachenko and Samsanov 1961; Coble 1970; Wilkinson and Ashby 1975; Arzt et al 1983; Nair and Tien 1987; Jagota and Dawson 1988; Christman et al 1989; Tvergaard 1990; Bao et al 1991; Broekenbrough et al 1991; Shi et al 1992; Dutta et al 1993; He et al 1993; Ravichandran 1994; Shen et al 1994) published so far, even though some of them are FEM-related, are restricted to unit cell-based methods in which the real structure is approximated to a periodic array of spheres, cylinders, cubes etc. These models are not applicable 
to cases where the spatial and the size distributions of different phases in any typical microstructure are complex and the material behaviour depends not only on the properties of the individual phases but also on their interactions. Therefore, in our numerical studies, we employed unit cells with representative microstructural geometries instead of regular ones. This allows us to exploit the FE method, which is particularly suited for handling complexities. This paper reviews briefly a few investigations carried out by the author to study the deformation behaviour of materials at microscopic scale.

Any analytical or numerical simulation involves three major aspects, namely, the geometrical representation of any structure, the material characteristics and the overall boundary conditions. In the following sections, we focus our attention on these three aspects.

\section{Microstructural geometry}

A microstructure observed at any particular location of a material is only a typical structure of the material at the microscopic scale and does not constitute an exact micro-building block. Therefore, for the purpose of simulation it becomes necessary to construct the representative microscopic geometries artificially, incorporating as many observed features as possible. In constructing such an artificial geometry one has to take into account the size distribution, shape, shape variation and volume fraction of the various phases present in the material. Since the FE mesh is generated only based on the relative dimensions of the various phases, the effect of the actual size of the particles or the phases can be incorporated not purely using the geometrical means but only in conjunction with the constitutive description. Even if the geometrical features observed at the sub-microscopic or atomistic scale are incorporated, a precise understanding of the material behaviour at these size scales is necessary and such a procedure may result in a prohibitively large number of finite elements costing large computer time and memory. Therefore, we incorporated the effect of size by choosing the material properties appropriate to the microscopic size-scale and the details are discussed in $\$ 3$. In the following paragraphs, we describe the procedure used for constructing the microstructural geometries in a few cases.

In order to model an aggregate of atomised powder (Ramakrishnan et al 1984; Ramakrishnan and Sivakumar 1990), a set of spheres (figure 1a) were chosen based on a $\log$ normal size distribution in the size range $25-250 \mu \mathrm{m}$ and placed in a rectangular box one by one, selecting each one randomly. The packing was done starting from the bottom row ensuring no overlapping of the spheres. Some minor adjustments of the positions of the spheres were then made for obtaining a locally stable configuration. The areal porosity of the configuration shown in figure $1 \mathrm{a}$ is 0.130 . The selected geometry was subdivided into a number of triangular and quadrilateral elements. By increasing the number of particles, it is possible to achieve a better representation of the size distribution of the particles and the positional randomness but it demands more computer memory and the CPU time. In addition, merely increasing the number of particles beyond an optimum limit may not improve the accuracy of the solution significantly. The rectangular box constructed to position these particles is only a fictitious one and does not represent 
any real container. This box is necessary for imposing the boundary conditions suitably. Although the particles considered in this study were of spherical shape, the method in general allows any shape.

In studies involving microstructures with two or more phases, it is necessary to take into account not only the size distribution of the second or the other phases but also their spatial distribution. For example, in the case of microstructures where the material is statistically homogeneous and isotropic, the distribution of the inclusion phases in the matrix phase need to obey spatial randomness. In order to ensure such a distribution, we employed the following procedure (Ramakrishnan and Arunachalam 1990; Ramakrishnan and Arunachalam 1993; Ramakrishnan et al 1991, 1996). It is well known that a perfectly random distribution of any phase results in identical area and volume fractions. We used the converse of this principle to establish the random distribution of any phase. For example, in the case of AlSiCp metal matrix composites (Ramakrishnan et al 1996), the particles were positioned at different locations in the matrix such that they appear random on a simple visual examination. Subsequently, this two-dimensional geometry was rotated about the $\mathrm{x}$ and $\mathrm{y}$ axes to generate cylindrical volumes with toroidal second phase particles and the volume fractions were computed. These volume fractions were compared with the area fraction. Depending on the difference, the second phase particles were relocated in the matrix so as to reduce the difference to a permissible level.

We used a master mesh (figure 2a) in the study of the two phase metal matrix composite (Ramakrishnan et al 1996), the porous material (Ramakrishnan and Arunachalam 1990, 1993) and the material exhibiting transformation induced plasticity (Ramakrishnan et al 1991). The master mesh comprises triangular and quadrilateral elements. A variety of microstructural morphologies can be modelled easily using the master mesh by assigning the material properties of a particular phase to selected elements. The matrix material properties are then assigned to the remaining elements. Using this procedure, the shape of the second phase as well as its volume fraction can be altered easily and two such cases are shown in figures $2 b$ and $2 c$.

\section{Material properties}

Since the values of the input-material properties considerably affect the simulation, adequate care must be exercised in using them, particularly the structure-sensitive ones because of their strong dependence on the size scale. The effect of the size scale can be realized by means of choosing the material properties appropriate to the size scale of interest and not merely by any geometrical means. If the size scale of the microstructure is at least an order of magnitude larger than the average grain size, data from simple tensile or compressive tests may be sufficient provided the true stress-strain variation is corrected for necking or bulging. The reliability can be further improved if the local material properties are determined by means of any FE simulation of the deformation of the tensile or the compression specimen itself and matching the numerically-established average properties to the experimentally-observed ones. As long as the material exhibits statistical homogeneity and isotropy in the size scale of interest, isotropic elastic modulii and von Mises constitutive flow behaviour can adequately and reliably describe the material 
deformation. However, for the size scales of the order of the grain size itself, the anisotropic modulii as well as the appropriate flow equations are to be used. For instance, to simulate crystalline slip deformation, shear stress based constitutive equations (Ramakrishnan and Atluri 1994; Ramakrishnan et al 1994) are more suitable.

In studying the deformation of multi-phase materials, it is necessary to consider not only the properties of the individual phases but also the effect of their interactions. For example, in analysing the deformation of metal matrix composites, merely considering the flow properties of the matrix and the particles does not describe the real case since the dislocation density in the matrix material depends on the size of the particles for a given local strain. The flow curves assumed for
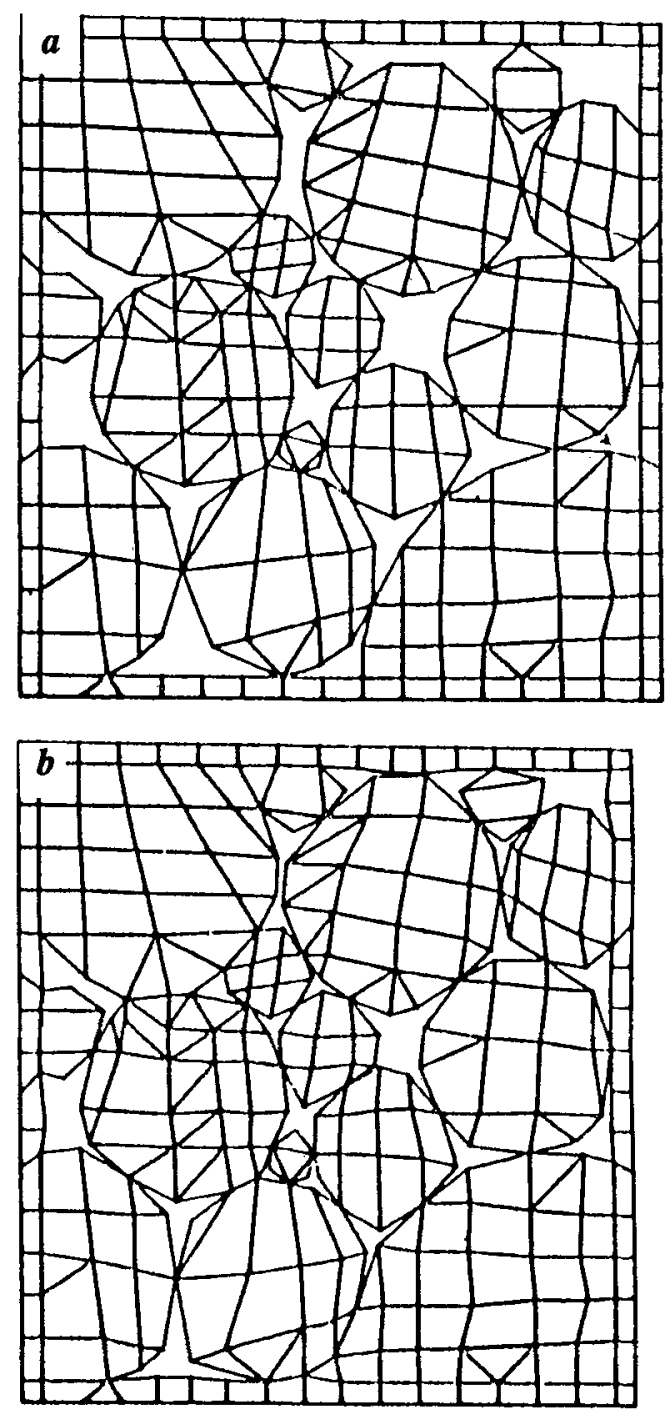

Figure 1. a-b. 

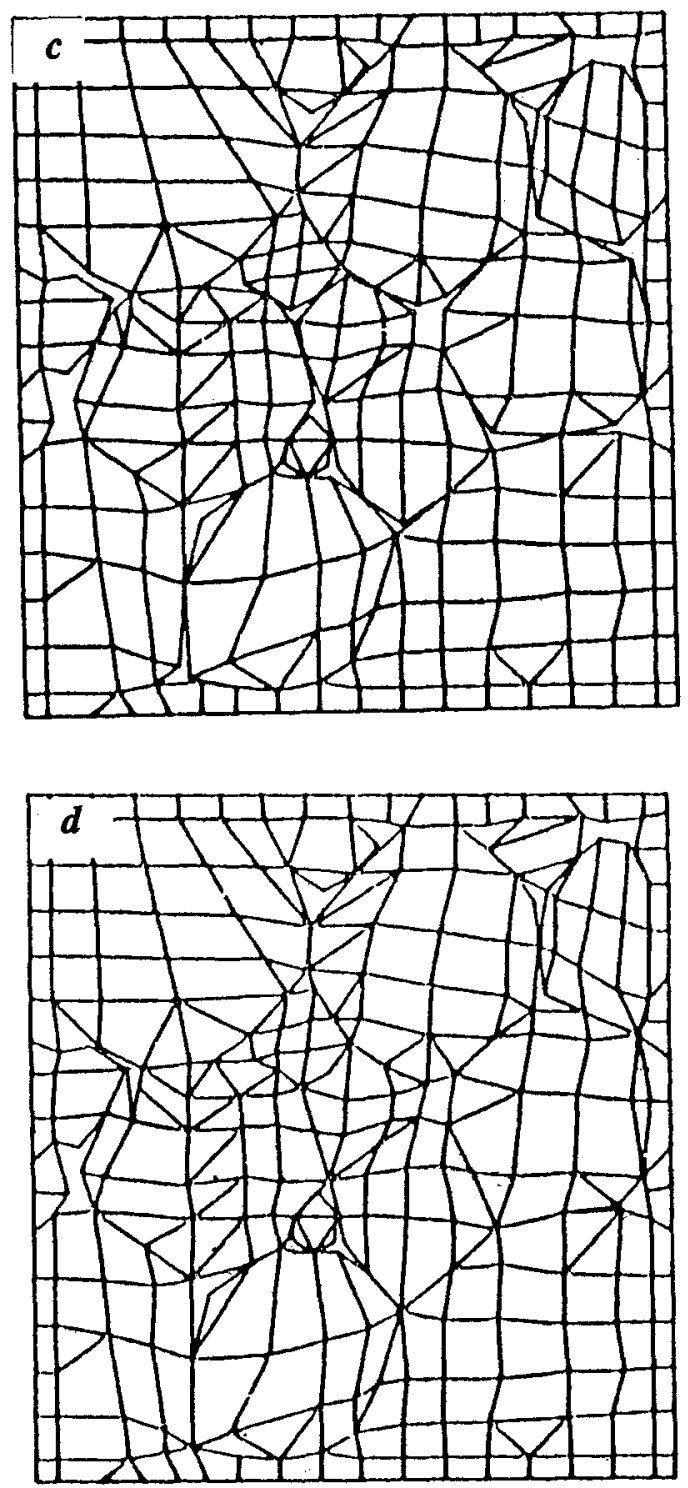

Figure 1. a-d. Delormalion scyuence of powder particles during HIPping.

characterizing the matrix material should accommodate such effects and these aspects are described in Ramakrishnan (1996).

There are a number of empirical as well as semi-empirical equations that take into account the effect of temperature and strain-rate. In our studies we used,

$$
\phi_{T}=\phi_{R T}+\left(\phi_{M T}-\phi_{R T}\right) \exp \left(-\alpha_{\phi} \zeta * * \beta_{\phi}\right)
$$

where $\zeta=\left(T_{M}-T\right) /\left(T-T_{R}\right)$, and $\phi$ represents properties such as modulus, Poisson's ratio, thermal conductivity, yield stress and coefficient of thermal expansion. 

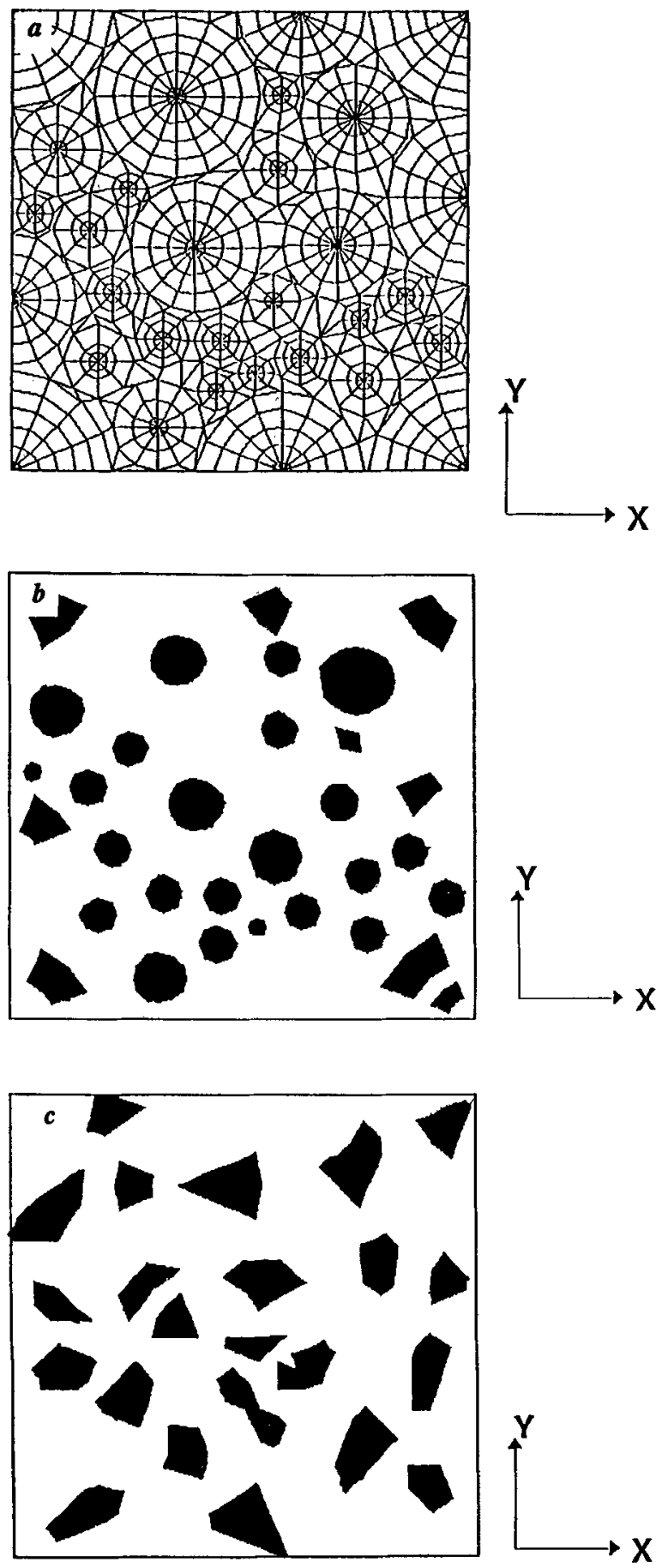

Figure 2. a. The master mesh for generating a variety of microstructural geometries; b-c. typical geometries generated using the master mesh (i) circular and (ii) angular second phase particles with a volume fraction of $20 \%$. 
Subscripts $R T, M T$ and $T$ denote room temperature, softening temperature and the temperature at which the property is to be computed respectively. $\alpha_{\phi}$ and $\beta_{\phi}$ are positive material constants which depend on the property $\phi$. While this empirical equation satisfies the properties at room temperature and the softening point $\left(\phi_{R T}\right.$ and $\phi_{M T}$ ) at $T_{R}$ and $T_{M}$ the property at any given temperature $T$ is determined as an interpolated value between $\phi_{R T}$ and $\phi_{M T}$. Modulii of any material remains virtually unaltered up to nearly half the softening temperature and steeply decreases beyond this temperature and the double exponential variation in (1) can capture such variations very accurately. For strain rate dependence, we used

$$
\phi / \phi_{0}=1+\eta \ln \left(\dot{\varepsilon} / \dot{\varepsilon}_{0}\right)
$$

where ' 0 ' denotes the reference state, $\eta$ a material constant and $\dot{\varepsilon}$ the strain rate. To describe the flow stress as a function of strain, we employed

or

$$
\begin{aligned}
& \sigma / \sigma_{y}=1+\kappa \varepsilon^{n}, \\
& \sigma / \sigma_{y}=A \varepsilon^{m}
\end{aligned}
$$

Since, neither of them can describe material softening, we used polynomial expressions for such requirements. The creep deformation is also characterized by the power-law relating the stress to strain rate. The material constants were established based on the various creep mechanisms which operate depending on the process parameters such as pressure and temperature and material parameters such as grain-size and diffusion coefficient. These aspects are described by Ramakrishnan et al (1984). Any deformation that arise as a result of allotropic transformation is simulated by assigning the stress-free strain developed during the transformation, which occurs above or below a critical stress or temperature, to the elements designated as the transforming phase.

Simulation of any crack propagation involves certain special features in the FE program. A crack is designated by a set of adjoining nodes and the crack is automatically propagated depending on the conditions set for propagation. A node which has the highest value of $\sigma_{\partial \theta} \sqrt{r}$ (where $r$ is the distance between the concerned node and the crack tip, and $\sigma_{\theta \theta}$ the stress perpendicular to the crack plane) from the set of nodes connected to the crack tip node, is selected first. Subsequently this node is broken into two nodes by assigning a new node address to the second one. The condition for the propagation is set based on the maximum of $\sigma_{\theta \theta} \sqrt{r}$ and not $\sigma_{\theta \theta}$ itself, in order to eliminate the effect of the finite size of the FE mesh. Also the programme should take care of the unloading and the redistribution of the stresses arising during each such step of the crack propagation.

\section{Boundary conditions}

The FE analysis of the deformation of any multi-phase material should be strictly three dimensional. However, any 3D analysis, in addition to being cumbersome, demands a large amount of computational resources. On the other hand, two 
dimensional approximations such as plane strain or plane stress are inadequate. As a compromise, we introduced a generalized plane strain method.

It is known that the plane strain or the plane stress deformation is modelled by setting the strain or the stress in the z-direction to zero respectively. In generalized plane strain methods a definite $z$-directional strain is imposed and the $z$-strain is chosen depending on the application. In the present type of study, we found it suitable to assign the value of the average strain in a particular constituent phase in the lateral direction ( $x$ or $y$ ) to the $z$-strain at any location of the phase. The details are discussed in Ramakrishnan et al (1996). If a homogeneous and isotropic material is loaded uniaxially, it produces identical strains in two lateral directions. Similarly any composite which is statistically homogeneous and isotropic also produces equal lateral global strains, even though the strain at any particular location depends on the microstructural morphology and the properties of the constituent phases. The generalized plane strain method discussed above forces the average strain in one lateral direction to be the same as that in the other lateral direction for each constituent phase independently. This not only ensures that the lateral strains are equal at the global level but also allows the $z$-strain to be different for different phases depending on their properties. For the volume fraction of the second phase approaching zero, the generalized plane strain condition approaches the plane stress condition as a limiting case.

Invariably the geometrical form of the representative microstructure was assumed to be of square shape in order to facilitate imposing the boundary conditions in $x$ and $y$ directions and cases such as particle assembly (figure 1) were contained in an artificial square box for the same reason. To ensure uniform deformation along any side of the square, we resorted to displacement or displacement-rate boundary conditions and not the nodal forces. Nevertheless, the nodal forces could be computed as an output of the FE analysis. Wherever the pressure or the forces were to be imposed directly, the required displacement boundary conditions were established iteratively (Ramakrishnan et al 1984).

\section{Salient results and discussions}

In many of our studies, FE was used essentially as a tool for simulating certain real experiments which are tedious or even difficult to perform. In some of the cases, our theoretical propositions could be cross-checked effectively using this method.

Using the simulation of the pressure sintering of powder particles it was possible to validate our theoretical expressions for the densification rate and the dependence of the densification rate on the effective stress (Ramakrishnan et al 1984). In any powder aggregate, the overall strength, sintering rate etc are directly related to the net contact area between the powder particles but the contact area cannot be easily measured. Since the porosity can be measured easily, the relationship between the porosity and the contact area established using analytical or numerical modelling aids in relating the effective material properties or the deformation behaviour of the powder aggregate to porosity, an experimentally determinable parameter. An analytical expression for the contact area between the particles (Ramakrishnan and Sivakumar 1990) in terms of the porosity was established and then validated using the FE simulation. 
In addition, the computer simulation provided a close study of the deformation of the powder particles (Ramakrishnan et al 1984). A typical sequence of the structures that evolve during pressure sintering of a set of powder particles, is shown in figures $1 \mathrm{a}-\mathrm{d}$. It can be seen that the fine particles and boundary regions of the coarse ones selectively deform and flow into the pores. Such an inhomogeneous deformation can lead to nucleation of new grains or strain-induced boundary migration in hot-pressed compacts. In fact, experimental observations show that the fine powder particles surrounding the coarse ones recrystallize and the new grains grow across the particle boundaries during HIPping and the results of the simulation conform to this observation. This aspect has an important bearing on the prior particle boundary problem in HIPped alloys.

In the case of the porous material (Ramakrishnan and Arunachalam 1990, 1993) and the particulate metal matrix composite (Ramakrishnan et al 1996), we used the master mesh for determining the effective elastic properties. There are analytical as well as experimental methods which can be used for predicting the variation of the elastic modulii of the porous compacts as a function of the porosity with a reasonable accuracy. However, an accurate determination of the effective Poisson's ratio $\left(v_{\mathrm{e}}\right)$ is not possible because of its large sensitivity to even small experimental errors. Many experimental investigations report only a qualitative trend of the variation of $v_{\mathrm{e}}$ with porosity. In this context, FE method becomes eminently suitable.

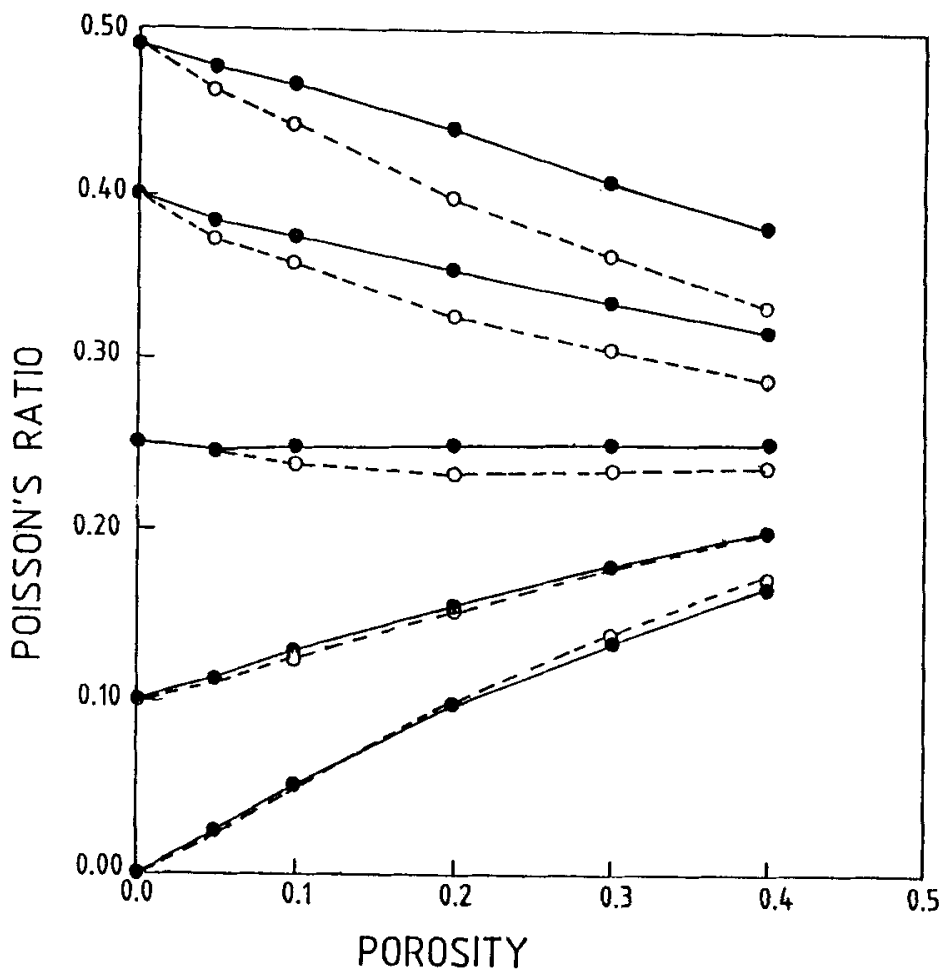

Figure 3. Variation of the effective Poisson's ratio of porous material with porosity (circular phase; - - , angular phase). 
We established the variation of $\nu_{\mathrm{e}}$ with porosity and the results are shown in figure 3. While $v_{e}$ for the materials with zero-porosity Poisson's ratio $\left(v_{0}\right)$ more than 0.25 decreases with porosity, increasing variation is seen for $v_{0}<0.25$. Interestingly, certain experimentally observed increasing trends of the effective Poisson's ratio with porosity, for materials such as $\mathrm{MgO}, \mathrm{SiO}_{2}$ etc for which $\nu_{0}<0.25$, were ignored by the earlier investigators as anomalous whereas the $\mathrm{FE}$ procedure allows a better understanding (Ramakrishnan and Arunachalam 1993).

We made an interesting observation that there is little effect of the shape of the second phase on the effective properties of MMCs as well as porous materials as long as the spatial and the orientation distribution of the second phase is random (Ramakrishnan and Arunachalam 1993; Ramakrishnan et al 1996), in other words, the material is statistically homogeneous and isotropic. Although, the stress gradients are steep adjacent to the sharp corners of the angular phase in contrast to that of the circular ones, the average global stresses are equal. In such cases, unit cell models based on regular geometries may predict a considerable effect of the shape of the second phase on the effective properties, since the periodic array of such unit cells does not correspond to statistically isotropic conditions. The normalized effective Young's modulus and the coefficient of thermal expansion of the MMC,

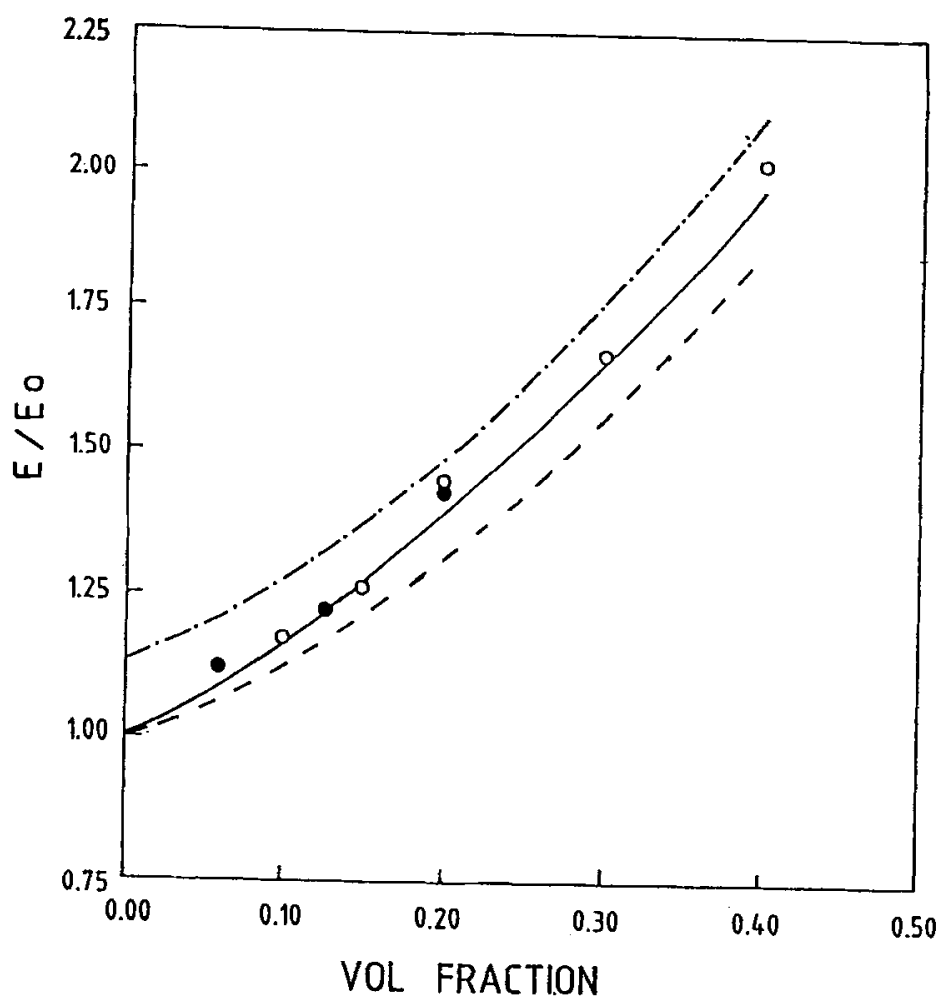

Figure 4. Effective Young's modulus of $\mathrm{Al}-\mathrm{SiCp}$ (MMC) vs volume fraction (- - - , plane strain condition; - - , plane stress condition; - - generalized plane strain condition and $O$, experimental data). 
determined using the simulation, are shown in figures 4 and 5 along with a few experimental data. The usefulness of the generalized plane strain method emerges clearly in these figures (Ramakrishnan et al 1996). In addition to the numerical studies, our analytical residual stress calculations in MMCs (Ramakrishnan 1996) helped us in quantitatively determining the relative importance of the matrix dislocation density and the load bearing effect of the second phase in strengthening MMCs.

For simulating the transformation-induced plasticity of $\mathrm{ZrO}_{2}-\mathrm{Al}_{2} \mathrm{O}_{3}$ (Ramakrishnan et al 1991), the properties of alumina were assigned to the elements of the matrix phase and those of zirconia to the circular or angular second-phase particles. The toughening process was simulated in steps. In the first step all the boundary nodes were set free except those required to be fixed to avoid rigid body modes. The residual stress developed because of the difference in the coefficients of thermal expansion (CTEs) of the matrix and the second phase was computed, stimulating the post fabrication cooling. This was followed by a search for the particle with the highest potential to transform and the dilatational transformation volume strain was imposed on all the elements falling in the particle. Only one particle was allowed to undergo transformation in each step since the compressive stress generated

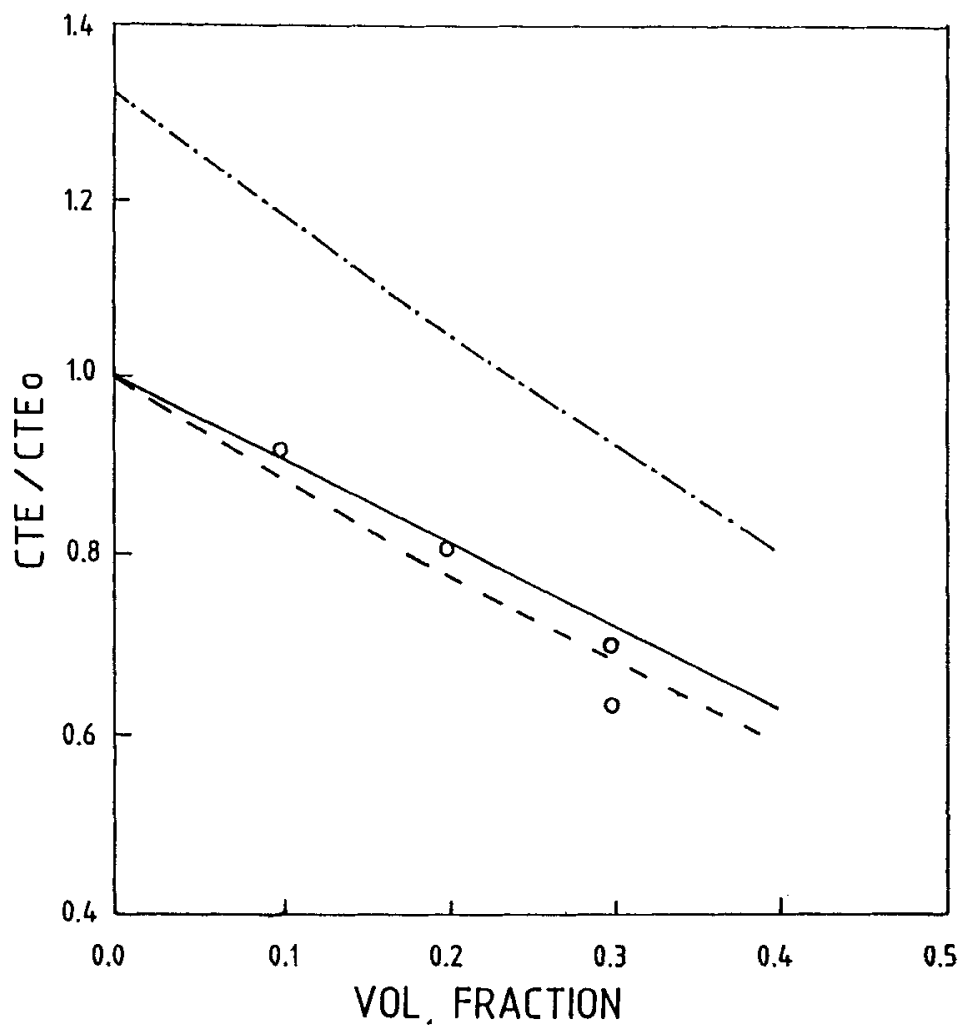

Figure 5. Effective thermal expansion coefïicient of $\mathrm{Al}-\mathrm{SiCp}(\mathrm{MMC})$ vs volume fraction (-.-.-, plane strain condition; - - - plane stress condition; - - generalized plune strain condition and $O$, experimental data). 
by the transformation of each particle precluded the transformation of the neighbouring ones. Such interactive effects cannot be captured using the conventional unit cell models of regular geometry. Using the procedure outlined above, we determined the constitutive behaviour of the transformation-induced plastic material (figure 6) and extended the analysis to studying the crack propagation characteristics in the material (figure 7). A close look at the serrated nature of the curves in figure 6 reveals that each kink is similar to the neighbouring ones and differs only in size. Each kink comprises of a vertical drop of the hydrostatic stress followed by a linear increase of this stress with a slope equal to the composite bulk modulus. The vertical drop is associated. with the compressive stress generated by the transforming particle, reducing the net far field tensile stress. From the lower-most state of this vertical drop, the far field stress is to be raised just to exceed the critical stress level of the next potential transforming particle and this process is continued. In a real situation, the serrations shrink as the number of the particles involved is much higher, resulting in a smooth constitutive curve. Increasing the volume fraction of the transforming phase improves the extent of the strain softening but does not appear to affect the modulus of the strain softening. Figure 7 compares the crack propagation in a transforming material to a nontransforming one. While the crack does not show any preference to any of the phases in a non-transforming

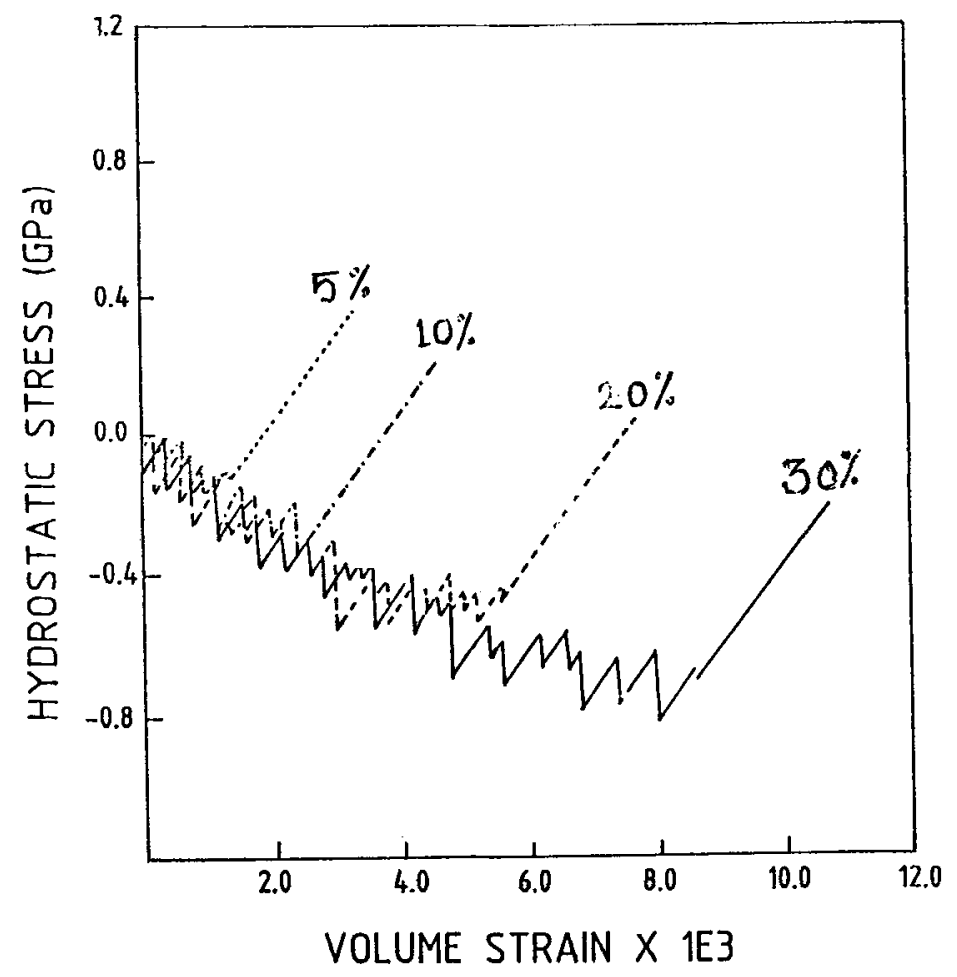

Figure 6. Effect of volume fraction of the second phase on the constitutive behaviour of $\mathrm{Al}_{2} \mathrm{O}_{3}-\mathrm{ZrO}_{2}$. 
material, in the transforming one the crack chooses to propagate through the matrix phase since the transforming phase and its neighbourhood experiences a strong compressive field. Using this simulation, it was possible to establish the increase in the fracture toughness of the material quantitatively.
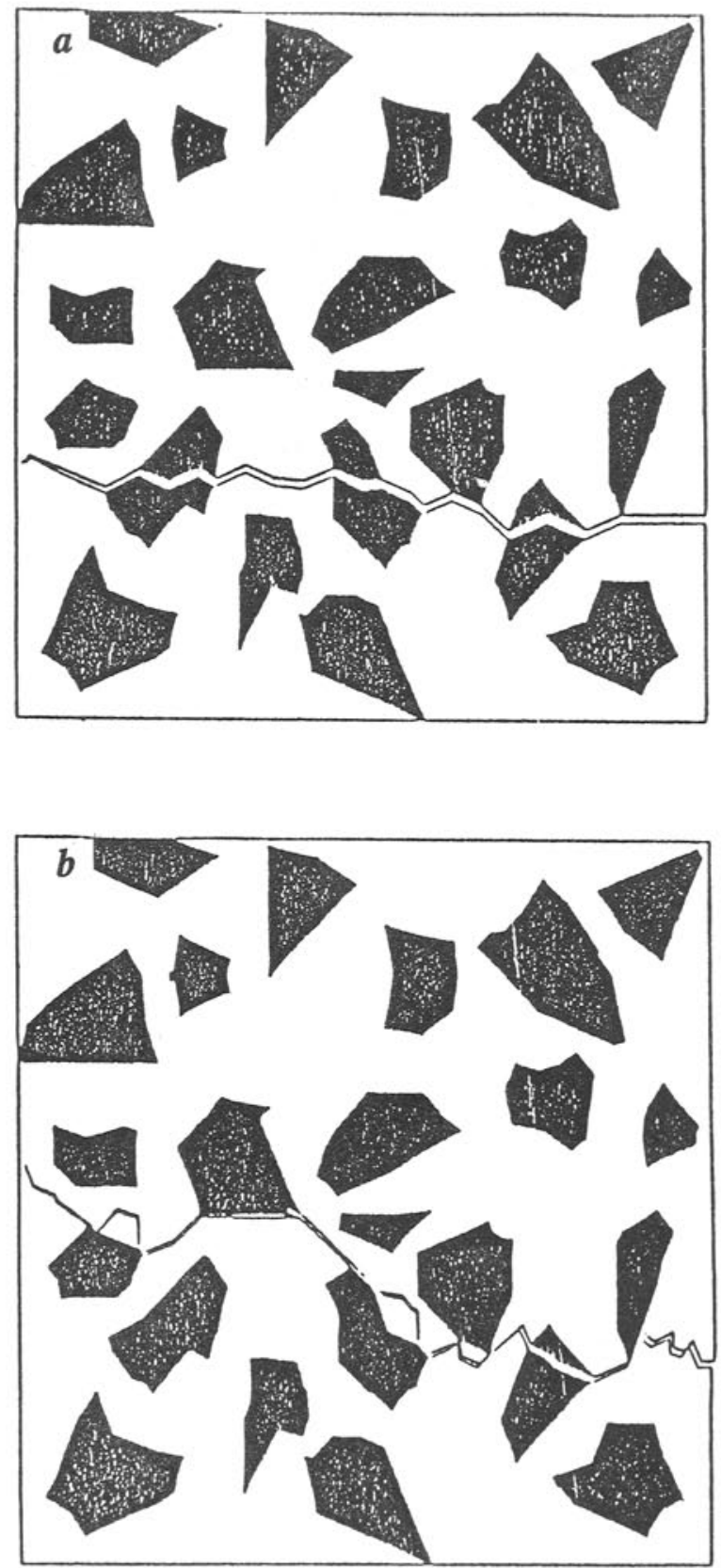

Figure 7. Crack propagation in (a) non-transforming matcrial and (b) transforming material. 
In an analysis of shear band formation in tensile or compressive specimens, we (Ramakrishnan and Atluri 1993, 1994; Ramakrishnan et al 1994) proposed a dual yield model where von Mises isotropic plastic flow and a directionally preferred shear flow occur concomitantly. This model enabled FE simulation of shear band as well as necking instabilities as shown in figure 8. Using this simulation it was possible to capture the microscopically-observed phenomenon of shear band formation across several grains without much deviation and could observe, what we termed as, kinematic docking of principal shear planes.

\section{Summary}

The overview presents a general finite element method for simulating the deformation at microscopic scale with a few illustrations from the following investigations

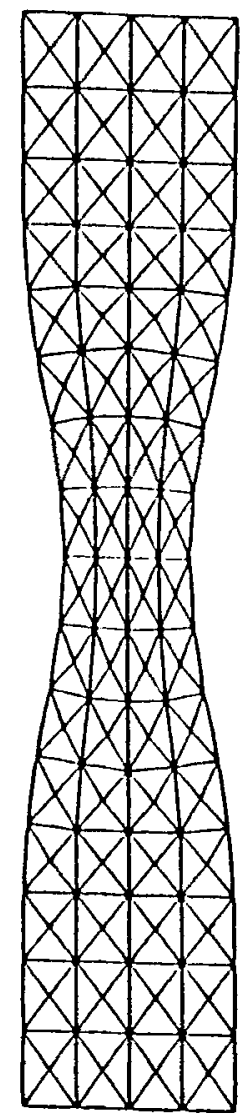

a

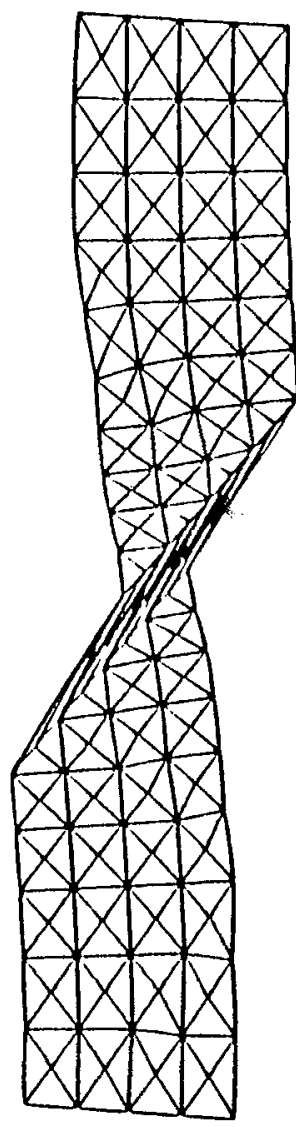

b

Figure 8. FE simulation of deformation in a plane strain convile specimen: (a) necking and (b) shear band formation. 
carried out earlier: (i) deformation of a set of powder particles during hot isostatic pressing; (ii) effective properties of a typical particulate metal matrix composite and porous material; (iii) constitutive behaviour of a material exhibiting transformation induced plasticity and (iv) shear band formation. A generalized plane strain approach to handle two dimensional approximation of what is essentially a three-dimensional deformation of microstructures along with a master mesh concept is presented. The investigations essentially cover the determination of the effective properties, computation of the stress and strain distributions, simulation of the crack propagation, shear band formation etc. In contrast to the unit cell methods based on regular geometries, the present method which is based on the actually observed microstructural geometries is more reliable in predicting the effective properties. In addition, this method accommodates more realistic and complex conditions compared to those supported by even well-explored analytical methods. Although we have considered only certain homogeneous and isotropic systems, this method can be extended easily to inhomogeneous and anisotropic microstructures as well.

Currently, the main limitation of this method is that the interfaces have been assumed to be perfectly bonded. Introducing the interfaces not only involves finer discretisation in the vicinity of the interfaces but also demands a rigorous description of the constitutive behaviour of the interface. Although the method is in its infancy presently, it shows a considerable scope for further refinement to accommodate more realistic conditions. It offers interesting possibilities for integrating the material development to component development and facilitating intelligent processing of materials.

\section{References}

Arzt E, Ashby M F and Esterling K E 1983 Metall. Trans. A14 211

Bao G, Hutchinson J W and McMeeking R M 1991 Acta Metall. 391871

Benvensite Y 1987 Mech. Mater. 6147

Broekenbrough J R, Suresh S and Wienecke H A 1991 Acta Metall. 39735

Budiansky B 1965 J. Mech. Phys. Solids 13223

Christensen R M 1990 J. Mech. Phys. Solids 38379

Christman T, Needleman A and Suresh S 1989 Acta Metall. 373029

Coble R L 1970 J. Appl. Phys. 414798

Dutta I, Sims J D and Seegenthaler D M 1993 Acta Metall. Mater. 41885

Hashin Z 1962 J. Appl. Mech. 29143

Hashin Z and Shtrikman S 1963 J. Mech. Phys. Solids 11127

He M Y, Evans A G and Curtin W A 1993 Acta Metall. Mater, 41871

Hill R 1965 J. Mech. Phys. Solids 13213

Jagota A and Dawson P R 1988 Acta Metall. 362551

Kovalachenko M S and Samsanov G V 1961 Poroshkov. Metall. 13

Mckenzie J K and Shuttleworth R 1948 Proc. R. Soc. B62 833

McLaughlin 1977 Int. J. Eng. Sci. 15237

Mori T and Tanaka K 1973 Acta Metall. 21571

Nair S V and Tien J K 1987 Metall. Trans. A18 97

Norris A N 1985 Mech. Mater. 41

Ramakrishnan N, Balakrishna Bhat T and Arunachalam V S 1984 Acta Metall. 32357

Ramakrishnan N and Sivakumar K 1990 Bull. Mater. Sci. 13217

Ramakrishnan N and Arunachalam V S 1990 J. Mater. Sci. 253930

Ramakrishnan N, Okada H and Atluri S N 1991 Acta Metall. Mater. 391297

Ramakrishnan N and Arunachalam V S 1993 J. Am. Ceram. Soc. 762745 
Ramakrishnan N and Atluri S N 1993 Mech. Mater. 17307

Ramakrishnan N and Atluri S N 1994 Int. J. Plasticity 10499

Ramakrishnan N, Okada $\mathrm{H}$ and Atluri S N 1994 Int. J. Plasticity 10521

Ramakrishnan N 1996 Acta Mater. 4469

Ramakrishnan N, Arun Kumar M and Radhakrishna Bhat B V 1996 J. Mater. Sci. 313507

Ravichandran K S 1994 Acta Metall. Mater. 421113

Shen Y -L, Finot M, Needleman A and Suresh S 1994 Acta Metall. Mater. 4277

Shi N, Wilner B and Arsenault J R 1992 Acta Metall. Mater. 42841

Tvergaard V 1990 Acta Metall. 38185

Wilkinson D S and Ashby M F 1975 Acta Metall. 231277

Zimmerman R W 1991 Mech. Mater. 1217 